\title{
Acrylonitrile-Styrene-Acrylate Particles with Different Microstructure for Improving the Toughness of Poly(styrene-co- acrylonitrile) Resin
}

\author{
Yuanying Zhang, Xuechun Zhang, Yongbin Cao, Jiachun Feng $\mathbb{D}$, and Wuli Yang \\ State Key Laboratory of Molecular Engineering of Polymers \& Department of Macromolecular Science, Fudan University, \\ Shanghai 200438, China
}

Correspondence should be addressed to Wuli Yang; wlyang@fudan.edu.cn

Received 21 July 2021; Accepted 26 August 2021; Published 11 September 2021

Academic Editor: Pierre Verge

Copyright () 2021 Yuanying Zhang et al. This is an open access article distributed under the Creative Commons Attribution License, which permits unrestricted use, distribution, and reproduction in any medium, provided the original work is properly cited.

\begin{abstract}
Herein, acrylonitrile-styrene-acrylate copolymer (ASA) particles with different microstructure were synthesized by emulsion polymerization and then used for toughening poly(styrene-co-acrylonitrile) (SAN) resin. The structure of ASA particles was confirmed by FTIR. TEM results demonstrated that the particles with different morphologies of multilobe shape, complete core-shell and dumbbell shape were obtained depending on the cross-linker amount. It was found that the toughening efficiency reached the highest when the ASA particles had complete core-shell structure and the shell composition was close to that of the SAN matrix. It was ascribed to the fact that the complete shell layer and similar shell composition provided sufficient interfacial adhesion and transferred stress to induce larger matrix deformation, so that the notched impact strength increased accordingly. Moreover, the notched impact strength of SAN/ASA blend was improved without significantly sacrificing tensile strength when adding $30 \mathrm{wt} \%$ ASA particles with the size of around $400 \mathrm{~nm}$. SEM results of the impactfractured surfaces revealed that irregular fluctuation and numerous microvoids occurred. It was deduced that the toughening mechanism was attributed to the crazings and cavitation of particles. Therefore, this study paved a way of toughening the resin by adjusting the microstructure of the particles including morphology, composition, and size.
\end{abstract}

\section{Introduction}

Poly(styrene-co-acrylonitrile) (SAN) is one of the most important thermoplastic materials fabricated by copolymerization of styrene (St) and acrylonitrile (AN), which has high rigidity, chemical and solvent resistance, and excellent processing properties [1-3]. However, poor toughness and notch sensitivity of SAN resin has led to its limited use [4, 5]. Therefore, improvement of its impact strength has been the research hotpoint. For example, chlorinated polyethylene (CPE) elastomer, which was an impact modifier for brittle materials, was melted with SAN resin, but it did not cause a drastic increase in impact strength or elongation at break because of the immiscibility between the two components [6]. What is more, CPE is prone to decompose at the processing temperature of SAN resin. Adding heat stabilizers and compatibilizers undoubtedly increases the cost and complexity of processing. Also, modified ethylene-propylene-diene terpolymer prepared by solution polymerization was introduced to toughen SAN resin [7, 8], a mass of solvent was used here, and the product conversion rate was low [9]. It was generally accepted that adding acrylonitrilebutadiene-styrene copolymer (ABS) was a successful and efficient way to toughen SAN resin $[10,11]$. Nevertheless, the double bonds $(\mathrm{C}=\mathrm{C})$ in polybutadiene $(\mathrm{PB})$ rubbery core of $\mathrm{ABS}$ particles are prone to deterioration and cross-linking due to oxygen, ultraviolet (UV), and other severe environmental circumstances, which results in poor aging resistance, weatherability, and antidiscoloration $[12,13]$.

It was well documented that the nanoparticles have been widely employed in toughening. For example, rubber nanoparticles were utilized to toughen the epoxy, and the effective 
means of energy dissipation were the cavitation of rubber nanoparticles and matrix plastic deformation [14]. Moreover, the particles with core-shell structure are desirable impact modifiers because of their unique composition, the rubbery core provides impact resistance even at low temperature, while the grafted shell serves as the compatibilizer [15, 16]. Wu et al. [17] synthesized three kinds of methyl methacrylate-butadiene-styrene (MBS) core-shell particles with different internal structures and found that MBS with "multilayer" structure improved the toughness of polyvinyl chloride (PVC) most significantly. Qian et al. [18] incorporated acrylonitrile (AN) monomer into the PMMA shell of poly(butadiene-co-styrene) (PBS) core/PMMA shell particles and controlled the particle dispersibility in the epoxy matrix by varying AN/MMA ratios to enhance the toughness.

Acrylonitrile-styrene-acrylate copolymer (ASA) particles with core-shell structure have been the effective impact modifiers. What is more, they have good weatherability and chemical resistance thanks to saturated acrylate unit [19]. Huang et al. tuned the particle sizes via controlling the feed modes and proportion of seed, and notched impact test results demonstrated that ASA particles with core phase of around $380 \mathrm{~nm}$ had the most remarkable toughening efficiency $[20,21]$. Tian et al. [22] investigated that ASA particles with $60 \mathrm{wt} \%$ PBA rubber core were the most suitable impact modifier for SAN resin, since it exhibited a good balance between toughness and rigidity. Furthermore, the influence of ASA particle addition amount, degree of crosslinking, and many other factors were fully discussed in others' work [23-26]. However, the effect of the microstructure of ASA particles on the toughening effect has not been thoroughly explored.

Herein, ASA particles with different microstructures, including different morphologies and shell compositions, have been synthesized by seeded emulsion polymerization. Furthermore, the influence of ASA particle microstructure on toughening SAN resin was fully investigated. It was found that the toughening efficiency reached the highest when the ASA particles had complete core-shell structure and the shell composition was close to that of SAN matrix, which could be attributed to that the complete shell layer and similar shell composition provided sufficient interfacial adhesion and transferred stress to induce larger matrix deformation, thus the notched impact strength increased accordingly. It was deduced that the toughening mechanism could be ascribed to the crazings and cavitation of particles.

\section{Materials and Methods}

2.1. Materials. Styrene (St, Aladdin Reagent Co.) and butyl acrylate (BA, TCI Co.) were purified by treating with silica gel column to remove the inhibitors. All other materials were used without further purification, including acrylonitrile (AN, TCI Co.), ethylene glycol dimethacrylate (EGDMA, J\&K Scientific Ltd.), allyl methacrylate (AMA, Aladdin Reagent Co.), sodium dodecyl sulfate (SDS, Aladdin Reagent Co.), potassium peroxydisulfate (KPS, Sinopharm Chemical Reagent Co., Ltd.), cumene hydroperoxide (CHP,
Aladdin Reagent Co.), ethylenediamine tetraacetic acid disodium salt (EDTA, Sinopharm Chemical Reagent Co., Ltd.), sodium hydroxymethanesulfinate dihydrate (SFS, TCI Co.), iron (II) sulfate heptahydrate (FES, Sinopharm Chemical Reagent Co., Ltd.), sodium hydrogen carbonate $\left(\mathrm{NaHCO}_{3}\right.$, Sinopharm Chemical Reagent Co., Ltd.), and magnesium sulfate anhydrous $\left(\mathrm{MgSO}_{4}\right.$, Shanghai Dahe Chemicals Co., Ltd.). Distilled deionized water (DDI) was provided by the laboratory of the author. Thermoplastic poly(styrene-coacrylonitrile) (SAN, PN107, Chimei Co., Ltd.), tris(2,4ditert-butylphenyl) phosphite (antioxidants 168, BASF China Co., Ltd.), and pentaerythritol tetrakis (3-(3,5-ditert-butyl-4-hydroxyphenyl) propionate) (antioxidants 1010, BASF China Co., Ltd.) were also purchased.

2.2. Preparation of ASA Particles. The ASA particles were prepared by seeded emulsion polymerization [27]. The synthesis observed three steps as follows, and the recipe was summarized in Table S1.

2.2.1. Preparation of $P B A$ Seed. Firstly, the PBA seed was prepared by batch process. Typically, $12 \mathrm{~g}$ of BA monomer, $0.096 \mathrm{~g}$ of SDS, $0.192 \mathrm{~g}$ of KPS, $0.1 \mathrm{~g}$ of EGDMA, and $90 \mathrm{~g}$ DDI were accurately weighed and added to a $250 \mathrm{~mL}$ three-neck glass reactor equipped with a reflux, a nitrogen purging inlet and outlet, and a mechanical stirrer. The reactor was held in an oil bath with thermostatic control. The reaction was carried out at $70^{\circ} \mathrm{C}$ and $240 \mathrm{rpm}$ for $6 \mathrm{~h}$.

2.2.2. Preparation of PBA Latex with Larger Particle Size. Afterwards, the preemulsion mixture consisting of $10 \mathrm{~g}$ of BA monomer, $0.025 \mathrm{~g}$ of the surfactant, $0.4-2.0 \mathrm{wt} \%$ crosslinker based on the total amount of the monomer, and $10 \mathrm{~g}$ of DDI was kept stirring for 40 min until it dispersed evenly and became milky white. The reaction was undergone in a $100 \mathrm{~mL}$ three-neck glass reactor with a reflux, a nitrogen purging inlet and outlet, and a mechanical stirrer. $5 \mathrm{~g}$ of the PBA seed emulsion prepared in the first stage, $0.08 \mathrm{~g}$ of the initiator, $0.03 \mathrm{~g}$ of $\mathrm{NaHCO}_{3}$, and $25 \mathrm{~g}$ DDI were added into the reactor. The preemulsion mixture was added by an injector with a feed rate of $12.5 \mathrm{mLh}^{-1}$. The reaction was carried out under the same aforementioned reaction condition.

2.2.3. Preparation of ASA Particles. Furthermore, ASA particles were prepared by a semicontinuous process. A certain amount of PBA latex with larger particle size and other ingredients including $24 \mathrm{mg}$ of SFS, $0.8 \mathrm{mg}$ EDTA, $0.2 \mathrm{mg}$ FES, $0.03 \mathrm{~g}$ of $\mathrm{NaHCO}_{3}$, and $26 \mathrm{~g}$ DDI were put into the $100 \mathrm{~mL}$ three-neck round bottom flask. The preemulsion mixture including $4 \mathrm{~g}$ St and AN monomers with different weight ratios (from $4: 1$ to $1: 1$ ), $0.01 \mathrm{~g}$ of surfactant (SDS), $8 \mathrm{mg}$ of oxidation initiator CHP, and $5 \mathrm{~g}$ DDI was stirred in order to disperse evenly. After the mixture became homogenous, it was injected into the reactor at the speed of $12.5 \mathrm{~mL} \mathrm{~h}^{-1}$. The polymerization was performed at $60^{\circ} \mathrm{C}$ and $240 \mathrm{rpm}$. After the polymerization for $6 \mathrm{~h}$, the temperature increased to $70^{\circ} \mathrm{C}$ for 1 hour in order to reduce residual monomer. 
2.2.4. Isolation of ASA Particles. Lastly, the ASA latexes were poured into the beakers at $80^{\circ} \mathrm{C}$ with a stirring speed of $400 \mathrm{rpm}, 3 \mathrm{wt} \% \mathrm{MgSO}_{4}$ aqueous solutions were prepared in $400 \mathrm{~mL}$ beakers as demulsifiers, and the pure ASA particles were obtained by filtration and dried under vacuum at $40^{\circ} \mathrm{C}$ for further use.

2.3. Preparation of SAN/ASA Blends. $45 \mathrm{~g}$ of SAN, $5-30 \mathrm{wt} \%$ ASA particles (based on SAN), and $0.2 \mathrm{wt} \%$ antioxidant 1010 and antioxidant 168 (based on SAN) were mixed by a HAAKE Polylab OS (Thermo Fisher Scientific Co., Ltd.) at $210^{\circ} \mathrm{C}$ and $20 \mathrm{rpm}$ for $8 \mathrm{~min}$.

\subsection{Characterization}

2.4.1. Particle Size and Size Distribution. The hydrodynamic size and polydispersity index (PDI) of PBA and ASA particles could be obtained directly by dynamic light scattering (Zetasizer Nano ZS90, Malvern Instrument Ltd.). The samples were diluted by distilled water in glass cuvettes to determine the size and PDI.

2.4.2. Morphology of ASA Particles. The morphology of ASA particles was recorded by a high contrast transmission electron microscope (TEM HT7800, HITACHI, Japan) at an accelerating voltage of $120 \mathrm{kV}$. The ASA particles were further diluted with ethanol and distilled water mixed medium $(1: 1)$, and then, diluted droplets were transferred onto the copper grids and dried at room temperature.

After ethanol and distilled water mixed medium $(1: 1)$ evaporation, ASA particles deposited on the silicon wafer were examined by a scanning electron microscope (SEM Ultra 55, Zeiss, Germany) equipped with a field-emission gun operated at $1.5 \mathrm{kV}$. All the surfaces were sprayed with a conductive layer of gold before scanning.

2.4.3. Fourier Transform Infrared Spectroscopy Analysis of ASA Particles. After grinding ASA samples with potassium bromide powder and pressing them into sheets, a Fourier transform infrared spectrometer (FTIR Nicolet 6700, Thermofisher, America) was employed to obtain infrared spectrum analysis.

2.4.4. Glass Transition Temperature $\left(T_{g}\right)$. The $T_{g}$ values were examined by a differential scanning calorimeter (DSC Q2000, TA, America). The temperature scanning range was -80 to $150^{\circ} \mathrm{C}$ at a heating rate of $20^{\circ} \mathrm{C} \mathrm{min}^{-1}$ under nitrogen atmosphere.

2.4.5. Gel Content and Grafting Degree of ASA Particles. The gel content and grafting degree were measured by the Soxhlet extraction method. $500 \mathrm{mg}\left(W_{0}\right)$ of dried ASA particles were weighed and extracted at $80^{\circ} \mathrm{C}$ for $12 \mathrm{~h}$ with $200 \mathrm{~mL}$ acetone as the extractor repeatedly. The remaining solids were dried under vacuum at $40^{\circ} \mathrm{C}$ and weighed $\left(W_{1}\right)$. The grafting degree $\left(G_{d}\right)$ is the weight ratio of grafted SAN to PBA core. The gel content and grafting degree were calculated as given in Equations (1) and (2); $A$ in Equation (2)

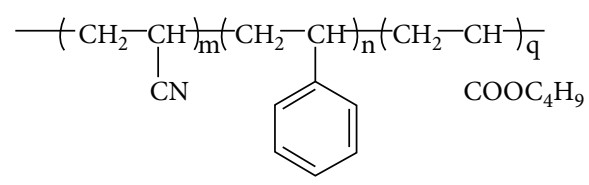

FIgURE 1: The chemical structure of the synthesized ASA particles.

TABle 1: A list of all of the ASA particles.

\begin{tabular}{|c|c|c|c|c|c|}
\hline Sample & $\begin{array}{c}\text { Cross-linker }^{\mathrm{a}} \\
(\mathrm{wt} \%)\end{array}$ & St: AN & $\underset{\mathrm{b}}{D_{h}}$ & $\mathrm{PDI}^{\mathrm{c}}$ & Morphology \\
\hline $\begin{array}{l}\text { ASA- } \\
\text { C1 }\end{array}$ & 0.4 & \multirow{5}{*}{$3: 1$} & 212 & 0.11 & Core-shell \\
\hline $\begin{array}{l}\text { ASA- } \\
\text { C2 }\end{array}$ & 0.8 & & 216 & 0.14 & $\begin{array}{l}\text { Complete core- } \\
\text { shell }\end{array}$ \\
\hline $\begin{array}{l}\text { ASA- } \\
\text { C3 }\end{array}$ & 1.2 & & 221 & 0.09 & Multilobes \\
\hline $\begin{array}{l}\text { ASA- } \\
\text { C4 }\end{array}$ & 1.6 & & 218 & 0.15 & Multilobes \\
\hline $\begin{array}{l}\text { ASA- } \\
\text { C5 }\end{array}$ & 2.0 & & 208 & 0.09 & Dumbbell \\
\hline $\begin{array}{l}\text { ASA- } \\
\text { R1 }\end{array}$ & \multirow{4}{*}{0.8} & $4: 1$ & 437 & 0.17 & \multirow{4}{*}{$\begin{array}{l}\text { Complete core- } \\
\text { shell }\end{array}$} \\
\hline $\begin{array}{l}\text { ASA- } \\
\text { R2 }\end{array}$ & & $3: 1$ & 433 & 0.04 & \\
\hline $\begin{array}{l}\text { ASA- } \\
\text { R3 }\end{array}$ & & $2: 1$ & 387 & 0.01 & \\
\hline $\begin{array}{l}\text { ASA- } \\
\text { R4 }\end{array}$ & & $1: 1$ & 370 & 0.01 & \\
\hline $\begin{array}{l}\text { ASA- } \\
\text { S1 }\end{array}$ & \multirow{3}{*}{0.8} & & 216 & 0.14 & \multirow{3}{*}{$\begin{array}{l}\text { Complete core- } \\
\text { shell }\end{array}$} \\
\hline $\begin{array}{l}\text { ASA- } \\
\text { S2 }\end{array}$ & & $3: 1$ & 337 & 0.04 & \\
\hline $\begin{array}{l}\text { ASA- } \\
\text { S3 }\end{array}$ & & & 433 & 0.04 & \\
\hline
\end{tabular}

${ }^{a}$ Based on the total amount of monomer in the fabrication process of PBA core. ${ }^{\mathrm{b}} D_{h}$, the hydrodynamic size is measured by Zetasizer Nano $\mathrm{ZS} 90 .{ }^{c} \mathrm{PDI}$, the polydispersity index of particles is measure by Zetasizer Nano ZS90.

represents the mass fraction of PBA core $[27,28]$.

$$
\begin{gathered}
G e l=\frac{W_{1}}{W_{0}} \times 100 \%, \\
G_{d}=\frac{W_{1} W_{0} \times A}{W_{0} \times A} \times 100 \% .
\end{gathered}
$$

2.4.6. Impact Property of SAN/ASA Blends. Standard specimens $\left(80 \times 10 \times 4 \mathrm{~mm}^{3}\right)$ with a V-type notch were fabricated through compression molding process by a certain mold and a notched prototype machine. Then, they were placed at ambient temperature for $24 \mathrm{~h}$ to eliminate residual stress. Notched Izod impact properties were carried out on an Izod impact tester (SS-3700CZD, Songshu Machine Factory, China) at room temperature according to Chinese standard GB/T 1843-2008. 


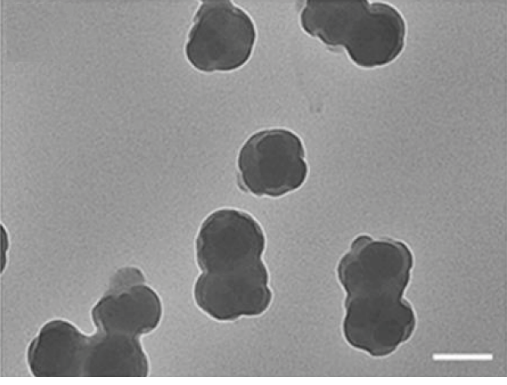

(a)

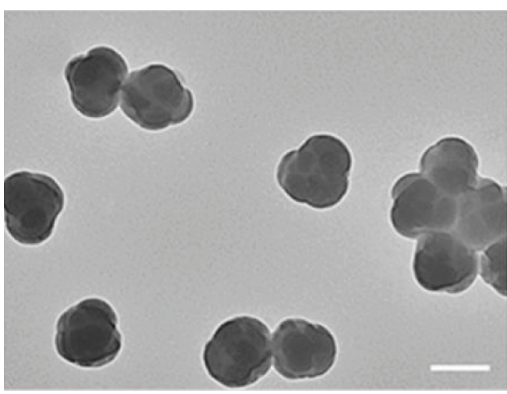

(c)

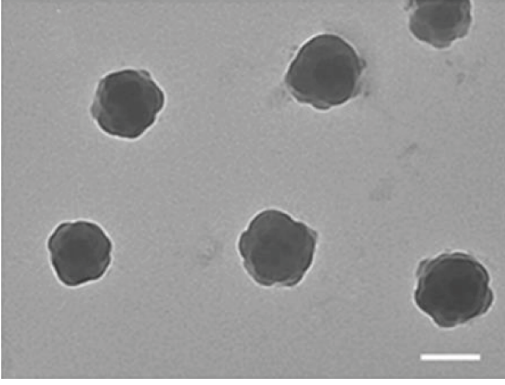

(b)

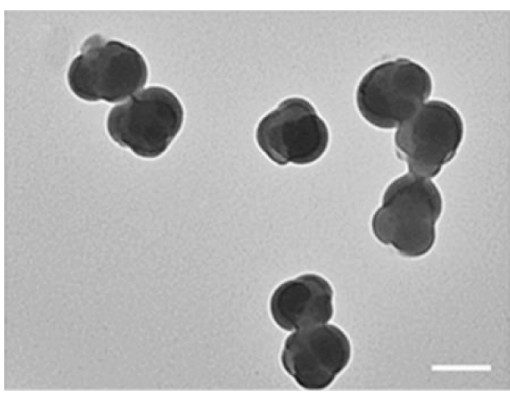

(d)

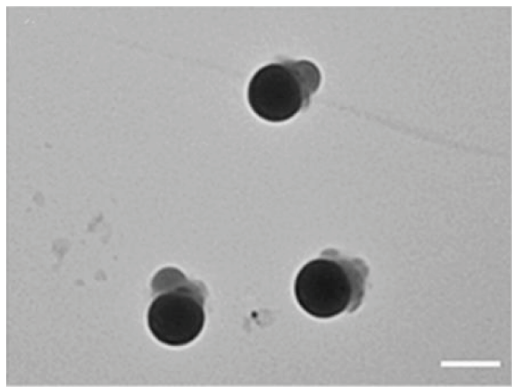

(e)

FIGURE 2: TEM images of the ASA particles with different morphologies prepared by different cross-linker amount: (a) ASA-C1 with 0.4 wt $\%$ cross-linker; (b) ASA-C2 with 0.8 wt $\%$ cross-linker; (c) ASA-C3 with $1.2 \mathrm{wt} \%$ cross-linker; (d) ASA-C4 with 1.6 wt\% cross-linker; (e) ASA-C5 with $2.0 \mathrm{wt} \%$ cross-linker. The scale bars represent $200 \mathrm{~nm}$.

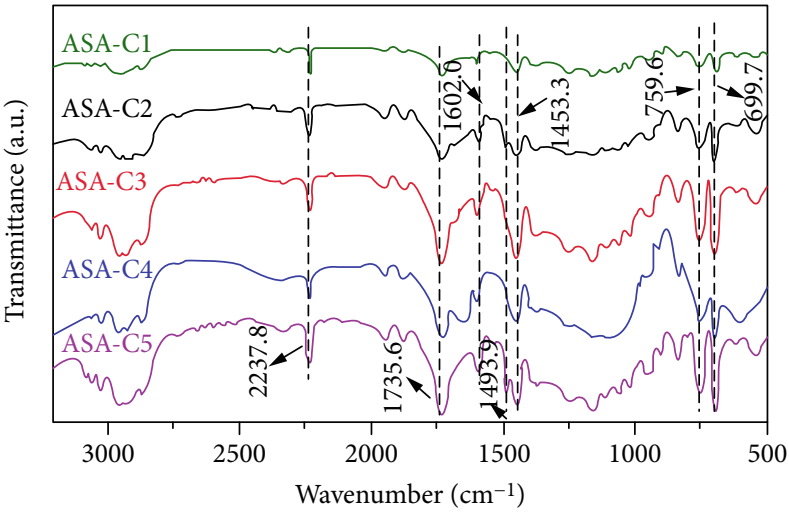

(a)

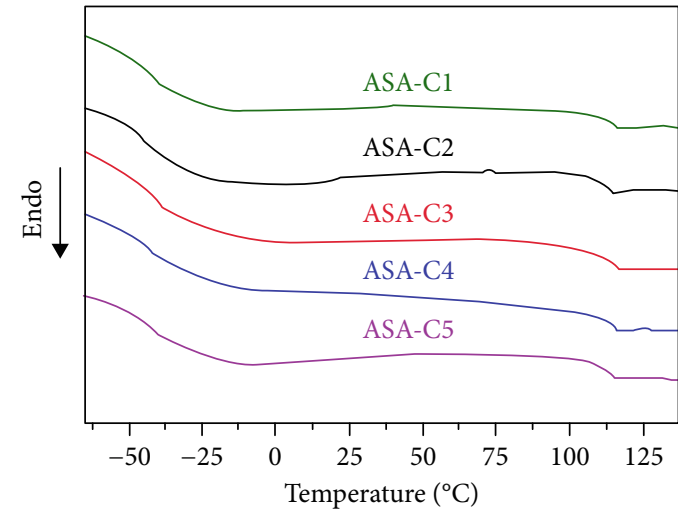

(b)

FIGURE 3: Fourier transform infrared spectrum (a) and differential scanning calorimetry curves (b) of ASA particles with different crosslinker amounts. 
2.4.7. Tensile Property and Modulus of SAN/ASA Blends. The dumbbell specimens were prepared by the compression molding process. The tensile property and modulus were carried out using a universal testing machine (Instron 5966, Instron, America) at room temperature with a stable rate of $5 \mathrm{~mm} \mathrm{~min}^{-1}$ for tensile tests according to Chinese standard GB/T 1040.2-2006.

\subsubsection{Morphology of Impact-Fractured Surface of SAN/ASA} Blends. The morphologies of impact-fractured surfaces were observed by scanning electron microscope (SEM Ultra 55, Zeiss, Germany) equipped with a field-emission gun operated at $5 \mathrm{kV}$. All the fracture surfaces were coated with an ultrathin conductive layer of gold before scanning.

\section{Results and Discussion}

3.1. Preparation of ASA Core-Shell Particles with Different Microstructure. In this work, a starved semicontinuous polymerization was utilized to prepare ASA particles in order to inhibit the generation of secondary particles to the utmost extent [21], and the relative amount of the shell phase to rubber core was 45/55 (wt/wt). During the seeded emulsion polymerization, EGDMA, together with the asymmetric cross-linker AMA was applied. The introduction of the allyl unit of AMA with comparatively low reaction activity paved the way for the following grafting process $[28,29]$. The chemical structure of the synthesized ASA particles is shown in Figure 1. The microstructure of ASA particles discussed in this part consisted of two aspects: one was the morphology and the other was shell composition.

3.1.1. Preparation of ASA Particles with Different Morphologies. By changing the amount of cross-linker from 0.4 to $2.0 \%$ in the PBA core, a series of uniformed ASA particles (ASA-C1-ASA-C5) of 200-220 nm were fabricated (Table 1, Table S2, and Figure S1). As was indicated in TEM images (Figure 2), the dark regions represented the soft PBA core, and the bright regions represented the hard SAN shell, respectively. It showed a trend of phase separation with the increasing amount of cross-linker from 0.4 to $2.0 \mathrm{wt} \%$. For the ASA particles (ASA-C1, ASA-C2) prepared by adding low content cross-linker (0.4 and $0.8 \mathrm{wt} \%$ ), core-shell structures were obvious (Figures 2(a) and 2(b)). Particularly for ASA particles (ASA-C2) with $0.8 \mathrm{wt} \%$ cross-linker, there existed a complete core-shell morphology, which meant that the cores were coated with the shells completely, with relatively few naked parts. ASA particles (ASA-C3, ASA-C4) with medium content crosslinker (1.2 and $1.6 \mathrm{wt} \%)$ emerged microdomains and formed the shape of multilobes (Figures 2(c) and 2(d)), which were the kind of kinetically frozen morphology [30]. Some parts of the core were naked, displaying deviation from complete core-shell structure. After further increasing the content of cross-linker to $2.0 \mathrm{wt} \%$, the morphology appeared like a dumbbell and most parts of the core were not covered by SAN shell, which meant that an apparent phase separation occurred (Figure 2(e)). With the increasing content of cross-linker, it became increasingly
TABLE 2: Glass transition temperature of ASA particles with different morphologies.

\begin{tabular}{lccc}
\hline Sample & Cross-linker $^{\mathrm{a}}(\mathrm{wt} \%)$ & $T_{g, 1}^{b}$ & $T_{g, 2}^{c}$ \\
\hline ASA-C1 & 0.4 & -46.9 & 111.7 \\
ASA-C2 & 0.8 & -46.6 & 111.9 \\
ASA-C3 & 1.2 & -45.1 & 112.4 \\
ASA-C4 & 1.6 & -45.2 & 112.2 \\
ASA-C5 & 2.0 & -45.6 & 111.5 \\
\hline
\end{tabular}

${ }^{a}$ Based on the total amount of monomer in the fabrication process of PBA core. ${ }^{\mathrm{b}} T_{g, 1},{ }^{\mathrm{c}} T_{g, 2}$, the glass transition temperature in low- and hightemperature regions is measured by DSC Q2000.

difficult for polymer chains to move and rearrange, and shell monomer and linear polymer chains exuded the particles to form new domains, resulting in phase separation to different extents [31,32].

Fourier transform infrared spectroscopy scanning from 500 to $3200 \mathrm{~cm}^{-1}$ was exploited to characterize the functional groups and verify the success in the synthesis of ASA particles. The apparent peak at $1735.6 \mathrm{~cm}^{-1}$ was the $\mathrm{C}=\mathrm{O}$ stretching vibration, representing the presence of PBA core (Figure S2). Besides, as is shown in Figure 3(a), the appearance of $\mathrm{C} \equiv \mathrm{N}$ absorption band at $2237.8 \mathrm{~cm}^{-1}$ gave strong supporting evidence that the SAN shell was successfully grafted onto the PBA core. The bands with a peak value at around $1602.0,1493.9$, and $1453.3 \mathrm{~cm}^{-1}$ were assigned to stretching vibration peak of the benzene ring skeleton. Besides, peaks at 759.6 and $699.7 \mathrm{~cm}^{-1}$ represented C-H bending or ring puckering $[20,33]$. All of the above evidence indicated the successful synthesis of ASA particles.

The DSC results indicated two $T_{g} s$ for all of the ASA particles, one was about $-45^{\circ} \mathrm{C}$ and the other was around $111^{\circ} \mathrm{C}$, corresponding to soft PBA cores and SAN shells, respectively (Figure 3(b) and Table 2), which fitted well with the core-shell structure model, further verifying the success in ASA particle synthesis [20].

Figure S3a illustrates how the content of cross-linker affected the gel content and grafting degree of ASA particles. When increasing the content of cross-linker from 0.4 to $1.2 \mathrm{wt} \%$, gel content showed an upward trend from approximately 57 to $74 \%$, and the grafting degree soared from $27 \%$ to nearly $67 \%$. It could be deduced that the cross-linker not only provided an appropriate crosslinking degree of the core but also offered the possibility of grafting shell layer. However, when adding $1.6 \mathrm{wt} \%$ crosslinker (ASA-C4), it appeared a slight downward trend, and with the further increase of cross-linker to $2.0 \mathrm{wt} \%$ (ASAC5), gel content and grafting degree both decreased sharply. It was mainly because of the phenomenon of phase separation. If the content of cross-linker was too high for the system, chain movement was restricted and grafting active sites were reduced.

3.1.2. Preparation of ASA Particles with Different Shell Compositions. ASA particles with different shell compositions were obtained by changing the feed ratio of St and 


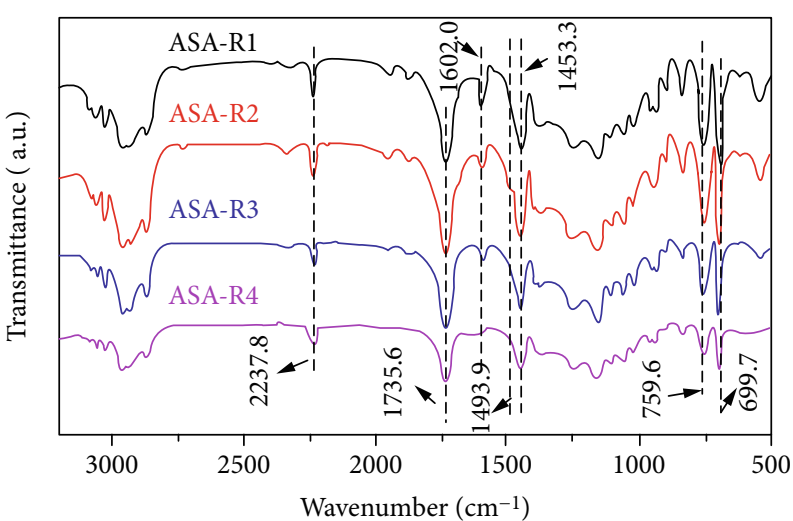

(a)

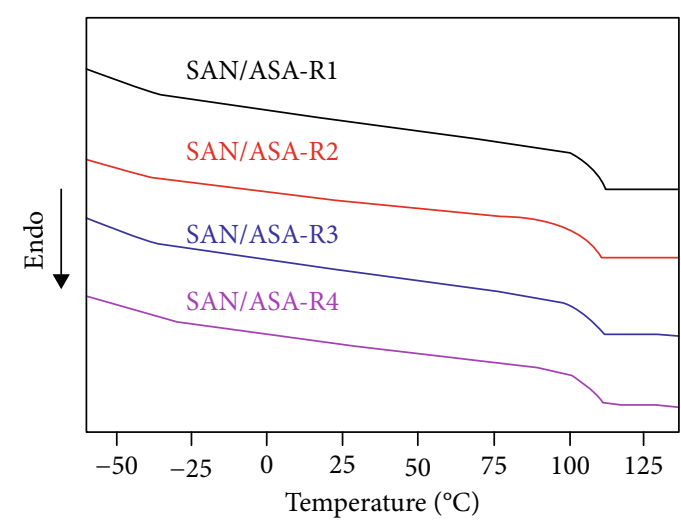

(b)

FIgURE 4: FTIR spectrum (a) and DSC result (b) of ASA particles with different shell compositions.

TABLE 3: Glass transition temperature of ASA particles with different shell compositions.

\begin{tabular}{lccc}
\hline Sample & Shell composition $^{\mathrm{a}}$ & $T_{g, 1}^{b}$ & $T_{g, 2}^{c}$ \\
\hline ASA-R1 & $4: 1$ & -43.9 & 111.6 \\
ASA-R2 & $3: 1$ & -42.5 & 112.6 \\
ASA-R3 & $2: 1$ & -42.1 & 117.0 \\
ASA-R4 & $1: 1$ & -40.9 & 117.7 \\
\hline
\end{tabular}

${ }^{\mathrm{a}}$ Based on shell monomer weight ratio (St:AN). ${ }^{\mathrm{b}} T_{g, 1},{ }^{\mathrm{c}} T_{g, 2}$, the glass transition temperature in low- and high-temperature regions is measured by DSC Q2000.

AN monomers during the second stage (Table 1 and Table S3). The particle sizes ranged from $370 \mathrm{~nm}$ to $437 \mathrm{~nm}$, and the values of PDI were all less than 0.2 , indicating that the size distribution was narrow. With the increasing proportion of acrylonitrile monomer, the hydrodynamic sizes decreased (ASA-R1 $\rightarrow$ ASA-R4) due to the appearance of secondary particles [34]. As was shown in TEM micrographs (Figure S4), all of this series of ASA particles had complete core-shell structure, and SEM images are given in Figure S5.

FTIR spectrum and DSC results reflected the variation of shell composition. In the infrared spectrum, the ratio of the peak area of the $\mathrm{C} \equiv \mathrm{N}$ stretching vibration (at $2237.4 \mathrm{~cm}^{-1}$ ) to the peak area of the benzene ring skeleton stretching vibration (at $1602.0 \mathrm{~cm}^{-1}$ ) increased as the proportion of acrylonitrile in the shell monomer increased (Figure 4(a)). From the DSC results, when the content of acrylonitrile increased, the glass transition temperature of SAN shell shifted to higher temperature (Figure 4(b) and Table 3 ). This phenomenon could be explained by the following Fox equation (3), which calculated the theoretical $T_{g}$ value of the SAN shell.

$$
\frac{1}{T_{g}}=\frac{W_{A}}{T_{g, A}}+\frac{W_{B}}{T_{g, B}} .
$$

$W_{A}$ and $W_{B}$ represented the proportion of AN and St in monomers, respectively; besides $T_{g}, T_{g, A}$ and $T_{g, B}$ signified the theoretical glass transition temperatures of SAN, PAN $\left(129^{\circ} \mathrm{C}\right)$, and $\mathrm{PS}\left(100^{\circ} \mathrm{C}\right)$, respectively [35].
Measured by the Soxhlet extraction method, as the amount of acrylonitrile increased, the grafting degree and gel content showed a trend of rising first and then falling simultaneously (Figure S3b). When the shell monomer ratio was $3: 1$, the feeding ratio was close to azeotropic point, and the copolymer composition was close to the feeding ratio, so the grafting degree and gel content correspondingly reached the maximum. Uniformed ASA particles with complete core-shell structure of different sizes were also fabricated successfully (Table 1, Table S4, Figure S6, and Figure S7).

\subsection{Effect of the Microstructure of ASA Particles on Toughening SAN Resin}

3.2.1. Influence of ASA Particle Morphology. In order to investigate the influence of ASA particle morphology on toughening SAN resin, samples containing $20 \mathrm{wt} \%$ ASA-C1 to ASA-C5 were examined by the notched Izod impact test. For complete core-shell structural ASA-C2 particles, the notched impact strength of blends firstly increased to $3.39 \mathrm{~kJ} \mathrm{~m}^{-2}$ from $3.02 \mathrm{~kJ} \mathrm{~m}^{-2}$ of SAN/ASA-C1 blends. However, for ASA-C3 and ASA-C4 with multilobe shape morphologies, the notched impact strength displayed a downward trend. When adding dumbbell-shaped ASA-C5, the notched impact strength was only $1.97 \mathrm{~kJ} \mathrm{~m}^{-2}$, which meant the toughening effect was not obvious (Figure 5(a)). Tensile strength was slightly lower than pure SAN resin, because of the low modulus of PBA core (Figure 5(b)).

Impact-fractured surfaces were obtained by SEM (Figure 6), the fractured surface of pure SAN resin was relatively smooth, and few crazes were observed, indicating SAN was a brittle material (Figure 6(a)). However, the whole surface became rougher as long as ASA particles were added. It appeared irregular fluctuation which represented the matrix deformation and numerous microvoids in its impactfractured surfaces (Figures 6(b)-6(f)), and the SEM of impact-fractured surfaces with smaller magnification showed lots of crazings like "scaly" patterns (Figure S8). The fractured surface of ASA-C2 was the roughest and the distribution of the voids was the most uniform, which distinguished it from other samples, exhibiting the highest 


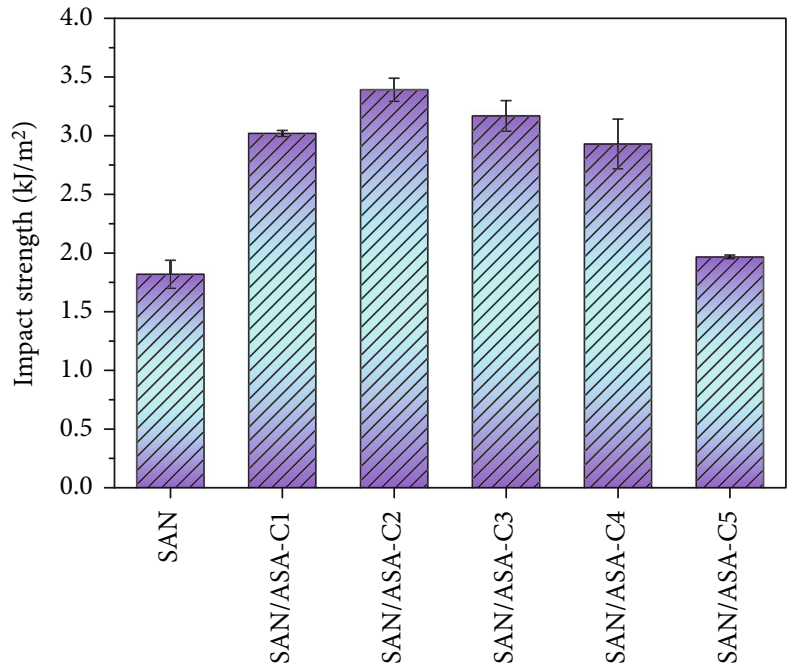

(a)

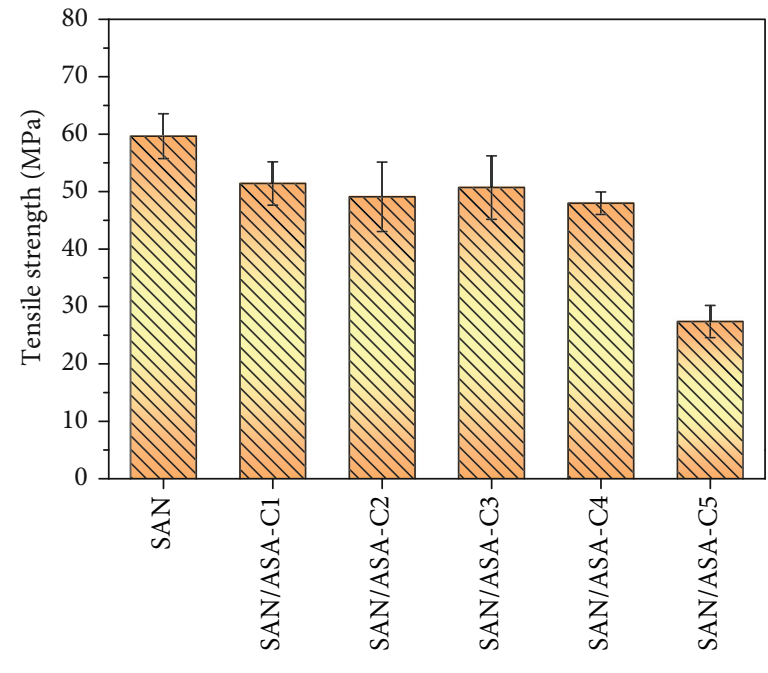

(b)

FIGURE 5: Effect of morphology of ASA particles on the notched Izod impact strengths (a) and tensile strengths (b) of SAN/ASA (100/20) blends. The error bars represent the standard deviation of measurements for 5 samples.

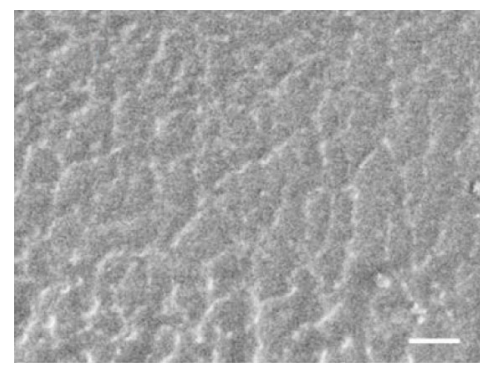

(a)

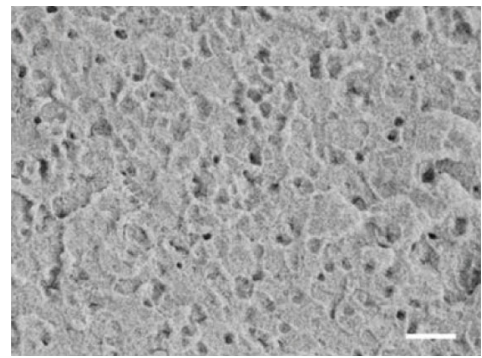

(c)

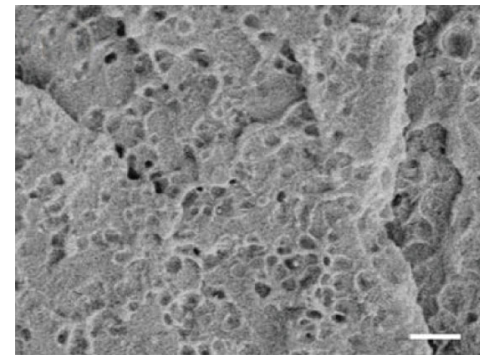

(e)

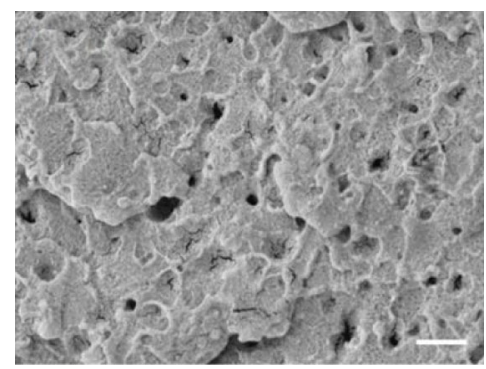

(b)

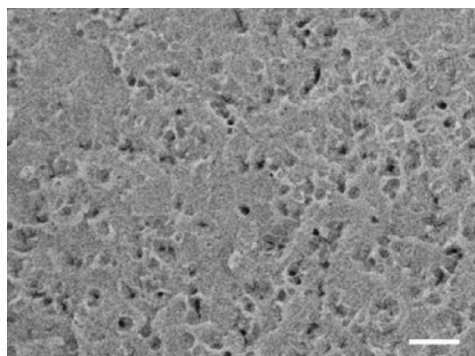

(d)

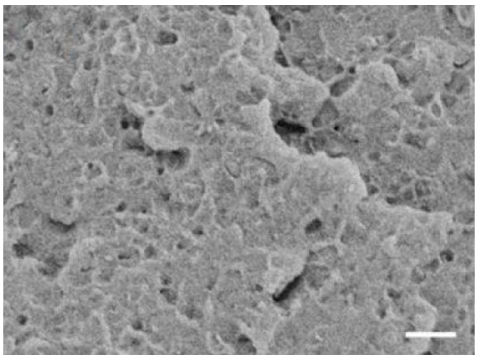

(f)

FIGURE 6: SEM images of the notched Izod impact-fractured surfaces for SAN/ASA blends with ASA particles of different morphologies: (a) pure SAN; (b) ASA-C1; (c) ASA-C2; (d) ASA-C3; (e) ASA-C4; (f) ASA-C5. The scale bars represent $1 \mu \mathrm{m}$. 


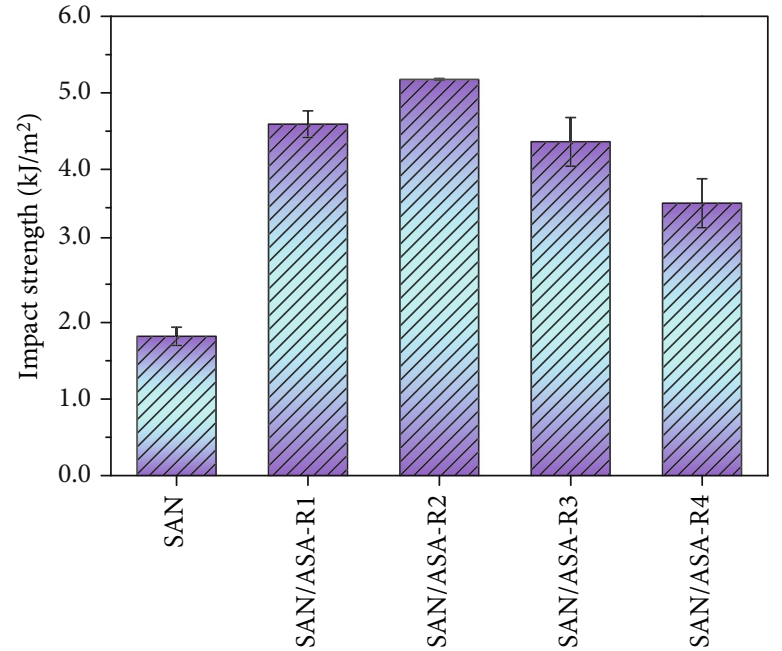

(a)

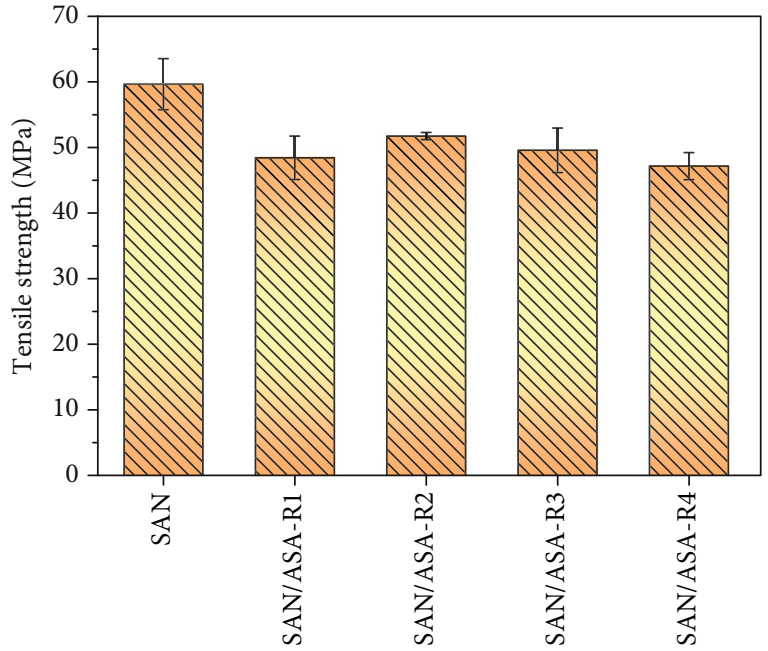

(b)

Figure 7: Effect of SAN shell composition on the notched Izod impact strengths (a) and tensile strengths (b) of SAN/ASA (100/30) blends. The error bars represent the standard deviation of measurements for 5 samples.

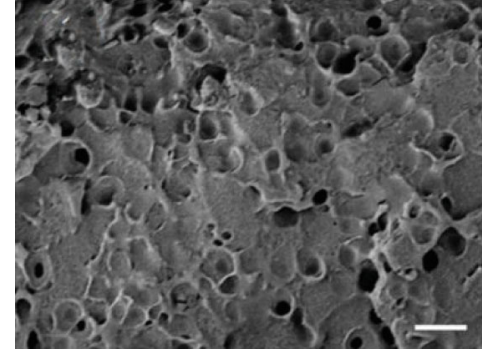

(a)

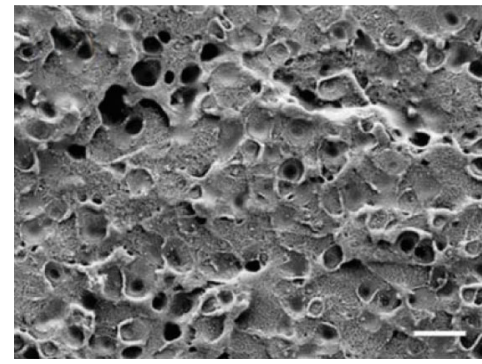

(c)

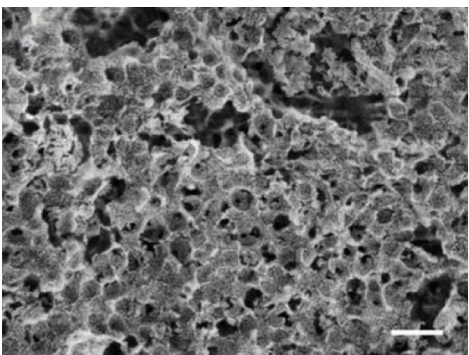

(b)

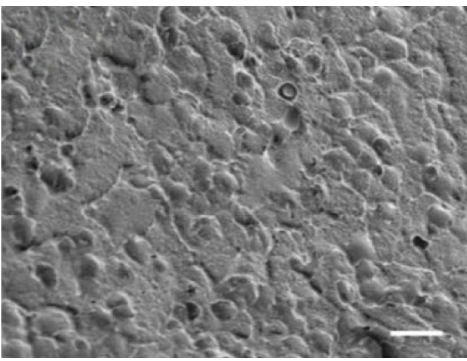

(d)

FIGURE 8: SEM images of the notched Izod impact-fractured surfaces for SAN/ASA (100/30) blends with ASA particles with different shell compositions: (a) ASA-R1; (b) ASA-R2; (c) ASA-R3; (d) ASA-R4. The scale bars represent $1 \mu \mathrm{m}$.

toughening efficiency (Figure 6(c)). All of the morphologies were correlated well with the aforementioned impact strength results. Therefore, complete core-shell particle morphology was crucial to toughen the resin matrix.

3.2.2. Influence of Shell Composition of ASA Particles. ASAR1-ASA-R4 particles with complete core-shell morphology and different shell compositions were blended with SAN resin in order to test the notched impact strength. During the test, SAN/ASA was kept to be $100 / 30$ (wt/wt). The results demonstrated that when the acrylonitrile content in shell composition decreased gradually, the notched impact strength of the blends showed a trend of increasing first and then decreased. Particularly when the ratio of St: AN was $3: 1$, the toughening effect was most significant, and the notched impact strength reached $5.18 \mathrm{~kJ} \mathrm{~m}^{-2}$ (Figure $7(\mathrm{a})$ ). The SEM photographs of impact-fractured surfaces of SAN/ASA blends are provided in Figure 8. Adding ASA-R2 particles (St : $\mathrm{AN}=3: 1$ ) made the fracture surface roughest, and numerous deformations in the matrix occurred (Figure 8(b)). In contrast, SAN/ASA-R4 blend demonstrated a relatively smooth impact-fractured surface, even a small part of rubber was trapped in microvoids, indicating poor interfacial adhesion (Figure 8(d)). Tensile strength displayed a slight downward trend (Figure $7(\mathrm{~b})$ ). 


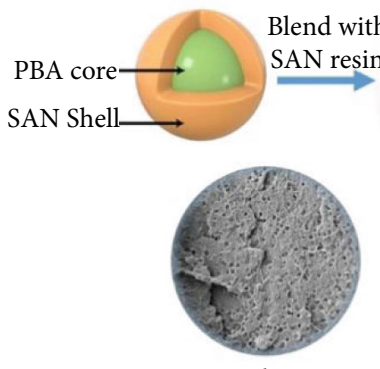

$5 \mathrm{k}$
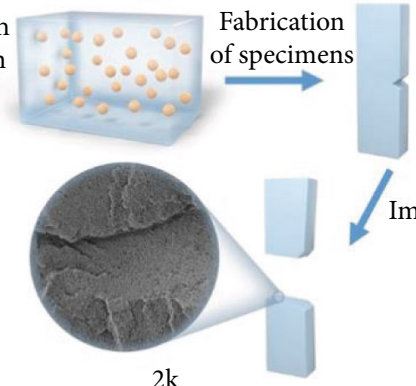

$2 \mathrm{k}$
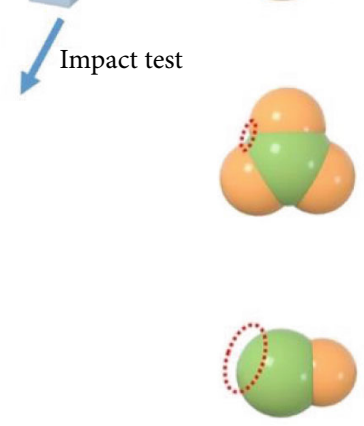

(a)

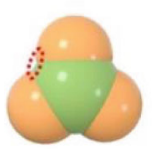

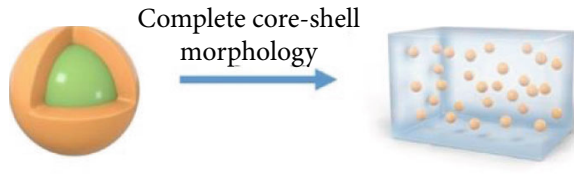

Multi-lobes morphology

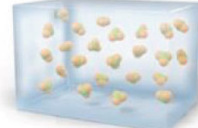

Dumbbell morphology

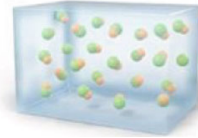

(b)
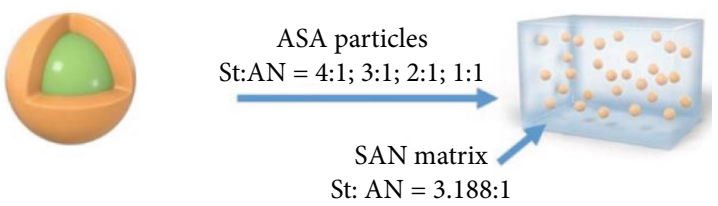

(c)

Scheme 1: (a) The preparation of ASA particles and their application as impact modifiers for SAN resin; (b) ASA particles with different morphologies for toughening SAN resin; (c) ASA particles with different shell compositions for toughening SAN resin.

Firstly, it was supposed that the toughening efficiency was related to integrity of core-shell structure [36]. The toughening mechanism mainly included the crazings and cavitation of rubber particles (Scheme 1(a)). For the ASA particles with core-shell structure, the rubbery core made the main contribution to toughening efficiency, while the rigid shell served as the "bridge" between SAN resin and the PBA cores to provide certain interfacial adhesion [37]. During the impact process, the ASA particles worked as the stress concentrators, the small deformation could create a high triaxial stress state in the rubber particles and then promoted cavitation, and impact energy was partly dissipated [38]. When ASA particles had a complete grafting shell layer (ASA-C2), the interfacial adhesion between matrix phase and soft cores was the most sufficient, which was beneficial for stress to transfer effectively between the two phases; thus, much more matrix volume participated in deformation and multiple crazing was initiated in SAN matrix to absorb impact energy (Scheme 1(b)). However, there existed a growing amount of naked rubber phase in multilobe shape morphology for ASA-C3 and ASA-C4 (red circles in Scheme 1(b)); the poor interfacial adhesion between the rubbery core and thermoplastic resin hindered the stress transfer, causing a limited toughening efficiency. However, the bad performance of SAN/ASA-C5 might ascribe to phase separation of ASA-C5 particles (Figure 2(e)). The more the uncovered rubber phase, the more the notched impact strength was reduced because of the increasing difficulty of stress transfer. Therefore, it could be deduced that the dumbbell shape of ASA particles was not beneficial to toughen SAN resin. Multilobe shape and dumbbell shape were less suitable to toughen SAN resin than complete core-shell structure because of their defects in the structure.

What is more, it could be concluded that the toughening effect was closely related to shell compositions. In the copolymerization system of St $\left(r_{1}=0.41\right)$ and AN $\left(r_{2}=0.04\right)$, the reactivity ratios $\left(r_{1}, r_{2}\right)$ were both less than 1 , and $r_{1} r_{2}<1$, so the azeotropic point condition is $\mathrm{F}_{1}=f_{1}=\left(1 r_{2}\right) /\left(2 r_{2} r_{1}\right)=$ 0.619 . The polymerization system was most stable by using the feed weight ratio of St: $\mathrm{AN}=3.188: 1$, so this feed ratio was adopted in industrial production. When the shell composition of ASA particles was St: $\mathrm{AN}=3: 1$, the shell monomer weight ratio was close to theoretical value (3.188), the shell can be compatible to SAN resin best from the theoretical perspective, which was beneficial to stress transfer to induce matrix deformation, so the ASA particles could exert its toughening effect to the utmost extent. However, when the feed ratio deviated from the azeotropic point condition, the notched impact strength decreased. Besides, the greater the deviation, the more pronounced the downward trend. Therefore, the toughening efficiency of ASAR1 (St : AN = 4:1) and ASA-R3 (St : AN = 2:1) was lower than that of ASA-R2 (St : AN = 3:1) but higher than that of ASA-R4 (St : AN = 1:1) (Scheme 1(c)).

3.3. Effect of Size and Content of ASA Particles on Toughening SAN Resin. ASA-S1 $(216 \mathrm{~nm})$, ASA-S2 $(337 \mathrm{~nm})$, and ASA-S3 $(433 \mathrm{~nm})$ with complete core-shell structure and similar shell composition were prepared for tests to study the influence of particle size on toughening efficiency. With the increasing diameter of particles, notched impact strength tended to rise and the void size in impact-fractured surface became larger (Figure 9(a) 


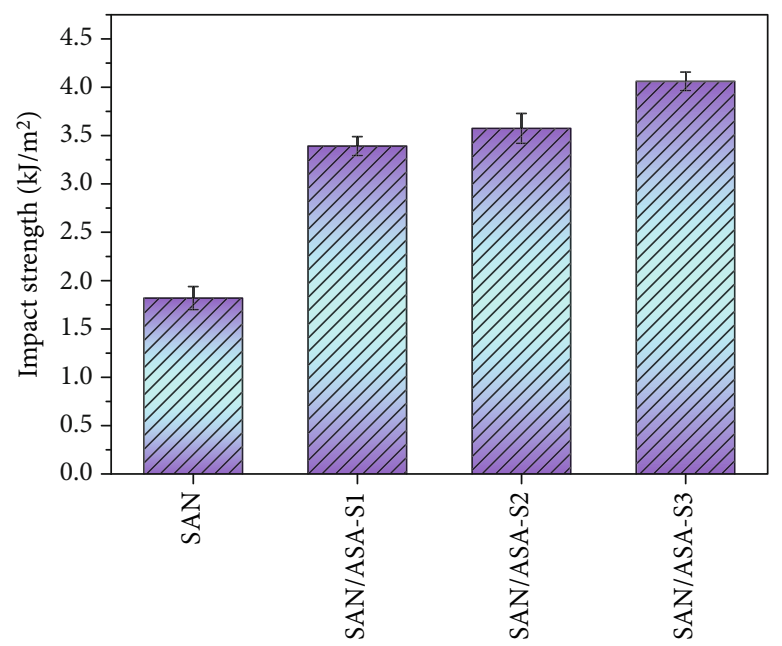

(a)

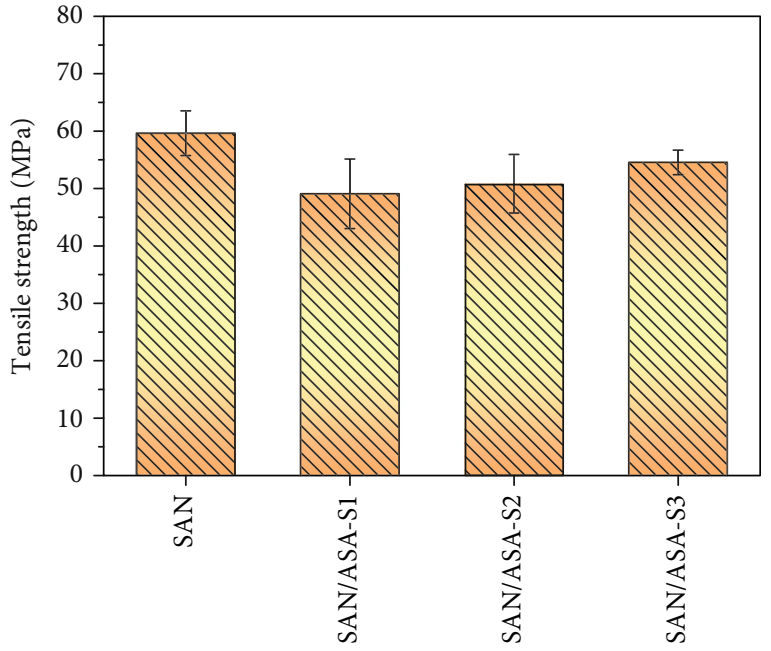

(b)

Figure 9: Effect of ASA particle sizes on the notched Izod impact strengths (a) and tensile strengths (b) of SAN/ASA (100/20) blends. The error bars represent the standard deviation of measurements for 5 samples.

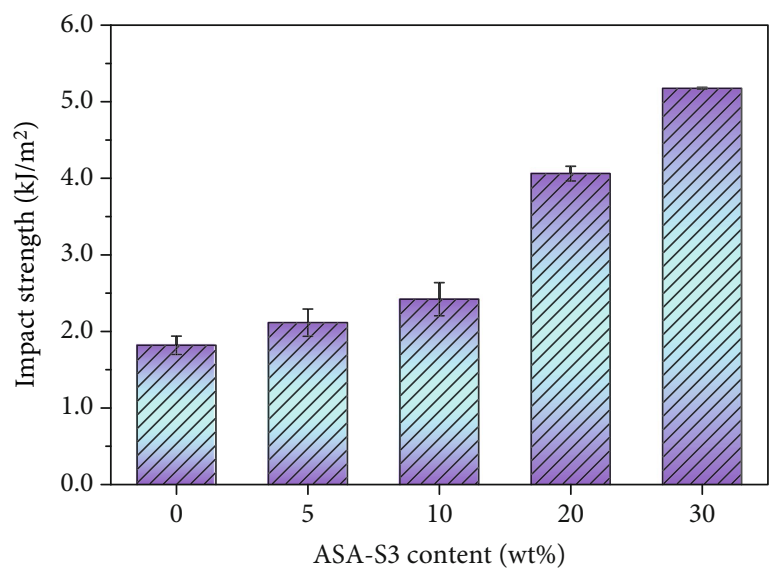

(a)

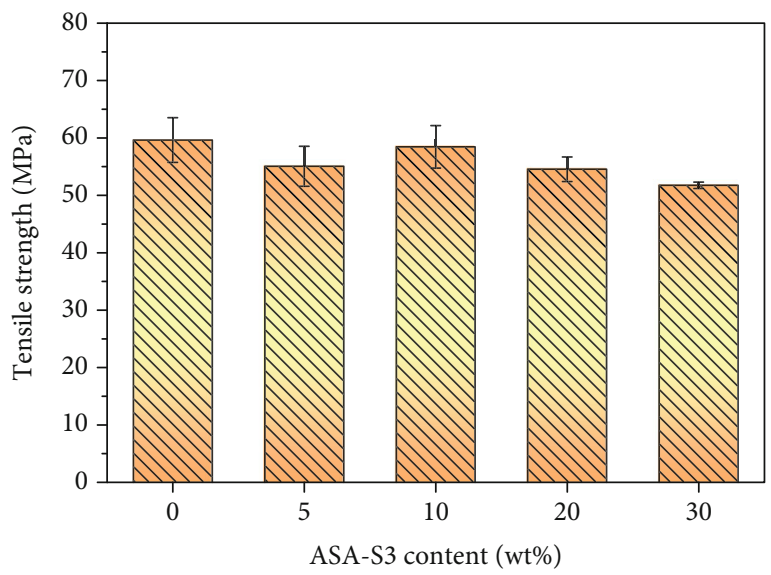

(b)

FIGURE 10: Effect of ASA particle (ASA-S3) content on the notched Izod impact strengths (a) and tensile strengths (b) of SAN/ASA blends. The error bars represent the standard deviation of measurements for 5 samples.

and Figure S9). It was obvious that particle size around $400 \mathrm{~nm}$ had a better toughening efficiency. The general mechanism of impact modifier mainly included crazing and cavitation of particles [38]. When the material was exposed to external force, stress concentration occurred in weak parts of the material, resulting in the phenomenon of crazing. If the particle size was too small, the crazing terminated too quickly, resulting in a low impact strength. However, ASA-S3 with larger particle size could not only initiate crazing but also control the growth of crazing, which absorbed plenty of energy $[39,40]$. The tensile strength of SAN/ASA-S3 was larger than the other two and a little bit lower than that of pure SAN resin (Figure 9(b)).

To study the influence of ASA particle content on toughening SAN resin, the sample ASA-S3 was selected. Figure 10(a) demonstrated that there was a steady increase when adding 5-10wt\% ASA particle. Once ASA particle amount was added to $20 \mathrm{wt} \%$, the impact strength was drastically improved. $30 \mathrm{wt} \%$ addition amount could toughen SAN resin more efficiently. With the increasing amount of ASA particles, more and more particles acted as stress concentrators to promote multiple crazings and plenty of voids to absorb energy [41]. In addition, the particle spacing decreased with the increasing content of ASA particles, and the surrounding stress field acted on each other, so that the deformation of matrix resin became drastic to absorb more impact energy [42]. Tensile strength was not sacrificed much with the addition of ASA particles according to Figure 10(b). Besides, the modulus of SAN/ASA (100/30) blend was $1666 \mathrm{MPa}$, which was only $15 \%$ lower than that of pure SAN resin, but the notched impact strength was $280 \%$ of SAN resin. SEM images of the impact-fractured surface are demonstrated in Figure S10. 


\section{Conclusions}

In summary, ASA particles with different morphologies and various shell compositions were prepared via seeded emulsion polymerization. It could be concluded that the microstructure, which consisted of the morphology and shell composition, was a key factor for ASA particles to toughen SAN resin. Complete core-shell structure of ASA particles led to best performance of toughening, while ASA particles with multilobe shape morphology had limited capability to toughen resin matrix, and ASA particles with dumbbell shape morphology could not toughen the SAN resin efficiently. What is more, when the monomer weight ratio in shell was close to that of SAN resin matrix composition, ASA particles could further exert their toughening efficiency. It was ascribed to the fact that the complete shell layer and similar shell composition provided sufficient interfacial adhesion and transferred stress to induce larger matrix deformation, so that the notched impact strength increased greatly. Besides, it was confirmed that ASA particles with reasonable diameter (around $400 \mathrm{~nm}$ ) showed advantage in toughening and $30 \%$ addition amount of ASA particles were more suitable to toughen than less addition amount. The impact-fractured surfaces revealed that the toughening mechanism was the crazings and cavitation of particles. This work provided new inspiration for the application of ASA particles to serve as a kind of impact modifier.

\section{Data Availability}

The data can be provided upon request to the corresponding author.

\section{Conflicts of Interest}

The authors declare that they have no conflict of interest.

\section{Acknowledgments}

This work was supported by the National Natural Science Foundation of China (Grant Nos. 51933002 and 51873041) and the National Key R\&D Program of China (Grant No. 2016YFC1100300).

\section{Supplementary Materials}

Supplementary Materials Table S1: the recipe for the preparation of ASA particles. Table S2: the preparation process of ASA particles with different morphologies. Table S3: change of size and size distribution during the preparation process of ASA particles with different shell monomer weight ratios. Table S4: the preparation process of ASA particles with different sizes. Figure S1: SEM images of the ASA particles with different morphologies prepared by different cross-linker amount: (a) ASA-C1 with $0.4 \mathrm{wt} \%$ cross-linker; (b) ASAC2 with 0.8 wt\% cross-linker; (c) ASA-C3 with $1.2 \mathrm{wt} \%$ cross-linker; (d) ASA-C4 with $1.6 \mathrm{wt} \%$ cross-linker; (e) ASA-C5 with $2.0 \mathrm{wt} \%$ cross-linker. The scale bars represent $200 \mathrm{~nm}$. Figure S2: Fourier transform infrared spectra of PBA core and ASA-C3. Figure S3: effect of cross-linker amount (a) and shell composition (b) of ASA particles on gel content and grafting degree. Figure S4: TEM images of ASA particles with different shell compositions: (a) ASAR1 with St: AN =4:1; (b) ASA-R2 with St: $A N=3: 1$; (c) ASA-R3 with St: AN = 2:1; (d) ASA-R4 with St: AN $=1: 1$. The scale bars represent $500 \mathrm{~nm}$. Figure S5: SEM images of ASA particles with different shell compositions: (a) ASA-R1 with St: AN $=4: 1$; (b) ASA-R2 with St : AN $=3: 1$; (c) ASA-R3 with St:AN = 2:1; (d) ASA-R4 with St $: A N=1: 1$. The scale bars represent $500 \mathrm{~nm}$. Figure S6: TEM images of the ASA particles with different sizes: (a) ASA-S1; (b) ASA-S2; (c) ASA-S3. The scale bars represent $500 \mathrm{~nm}$. Figure S7: SEM images of the ASA particles with different sizes: (a) ASA-S1; (b) ASA-S2; (c) ASA-S3. The scale bars represent $200 \mathrm{~nm}$. Figure S8: SEM images of the notched Izod impact-fractured surfaces with smaller magnification for SAN/ASA blends with ASA particles of different morphologies: (a) pure SAN; (b) ASA-C1; (c) ASA-C2; (d) ASA-C3; (e) ASA-C4; (f) ASA-C5. The scale bars represent $5 \mu \mathrm{m}$. Figure S9: SEM images of the notched Izod impactfractured surfaces for SAN/ASA (100/20) blends with ASA particles with different sizes: (a) pure SAN; (b) ASA-S1; (c) ASA-S2; (d) ASA-S3. The scale bars represent $1 \mu \mathrm{m}$. Figure S10: SEM images of the notched Izod impact-fractured surfaces for SAN/ASA blends with ASA-S3 with different contents: (a) $5 \mathrm{wt} \%$; (b) $10 \mathrm{wt} \%$; (c) $20 \mathrm{wt} \%$; (d) $30 \mathrm{wt} \%$. The scale bars represent $1 \mu \mathrm{m}$. (Supplementary Materials)

\section{References}

[1] S. Wacharawichanant, N. Thongbunyoung, P. Churdchoo, T. Sookjai, and S. Thongyai, "Morphology and properties of poly(styrene-co-acrylonitrile)/poly(methyl methacrylate)/zinc oxide composites," Journal of Reinforced Plastics and Composites, vol. 32, no. 15, pp. 1112-1121, 2013.

[2] M. Ashok, S. Deepika, P. Sowndharya, and K. Muthukumar, "Cotton candy driven chitosan and gelatin coated poly(styrene-co-acrylonitrile) microfibers for anti-microbial wound dressing applications," Materials Research Express, vol. 6, no. 12, article 125339, 2019.

[3] K. Bedjaoui, R. Krache, A. Marcos-Fernandez, and M. Guessoum, "The effect of compatibilizer sebs on the mechanical, morphological and thermal properties of the polystyrene/poly (styrene-co-acrylonitrile) copolymer blends," Materials Research Express, vol. 6, no. 10, article 105334, 2019.

[4] J. Scheirs and D. Priddy, Modern Styrenic Polymers: Polystyrenes and Styrenic Copolymers, John Wiley \& Sons, 2003.

[5] Y. P. Zhu, L. S. Wang, A. Q. Zhang, X. Wang, T. Cai, and $\mathrm{X}$. Zeng, "Toughening effect of poly(ethene-co-1-butene)graft-methyl methacrylate and acrylonitrile on styreneacrylonitrile copolymer (SAN)," Journal of Macromolecular Science, Part B, vol. 49, no. 4, pp. 757-769, 2010.

[6] J. S. Wang, Y. Ding, Y. Yu, G. N. Li, and F. Sun, "Influence of composition and synthesis conditions on microstructure and properties of acrylonitrile-chlorinated polyethylene-styrene copolymer," Journal of Applied Polymer Science, vol. 130, no. 3, pp. 2136-2142, 2013.

[7] Z. Zeng, L. Wang, T. Cai, and X. Zeng, "Synthesis of high rubber styrene-EPDM -acrylonitrile graft copolymer and its 
toughening effect on SAN," Journal of Applied Polymer Science, vol. 94, no. 2, pp. 416-423, 2004.

[8] W. Liu, L. Wang, J. Mai, and A. Zhang, "Synthesis of EPDM-gMAN by suspension graft copolymerization and its toughening effect on SAN resin," Polymer Bulletin, vol. 71, no. 12, pp. 3111-3129, 2014.

[9] J. Mai, J. Zhu, and L. Wang, "Toughening effect of ethylene propylene dieneterpolymer-graft-styrene-acrylonitrile copolymer (EPDM-g-SAN) on SAN resin," Journal of Macromolecular Science, Part B, vol. 53, no. 3, pp. 513-528, 2014.

[10] J. Zhu, Y. Hu, Y. Tang, and B. Wang, "Effects of styreneacrylonitrile contents on the properties of ABS/SAN blends for fused deposition modeling," Journal of Applied Polymer Science, vol. 134, no. 7, article 44477, 2017.

[11] M. A. Dundar, G. S. Dhaliwal, and E. Ayorinde, "Experimental and numerical investigation on flexural behavior ofacrylonitrile-butadiene-styrenepolymer," Polymer Engineering and Science, vol. 60, no. 11, pp. 2930-2944, 2020.

[12] C. K. Radhakrishnan, R. Alex, and G. Unnikrishnan, “Thermal, ozone and gamma ageing of styrene butadiene rubber and poly(ethylene- co -vinyl acetate) blends," Polymer Degradation and Stability, vol. 91, no. 4, pp. 902-910, 2006.

[13] A. Gottschalk and C. Bonten, "Styrene copolymers," Kunststoffe-Plast Europe, vol. 95, no. 10, 2005.

[14] B. T. Marouf, Y.-W. Mai, R. Bagheri, and R. A. Pearson, "Toughening of epoxy nanocomposites: nano and hybrid effects," Polymer Reviews, vol. 56, no. 1, pp. 70-112, 2016.

[15] Y.-J. Huang, J.-H. Wu, J.-G. Liang, M.-W. Hsu, and J.-K. Ma, "Toughening of unsaturated polyester resins with core-shell rubbers," Journal of Applied Polymer Science, vol. 107, no. 2, pp. 939-950, 2008.

[16] Y. Zhu, X. Gao, and Y. Luo, "Core-shell particles of poly(methyl methacrylate)-block-poly(n-butyl acrylate) synthesized via reversible addition-fragmentation chain-transfer emulsion polymerization and the polymer's application in toughening polycarbonate," Journal of Applied Polymer Science, vol. 133, no. 1, article 42833, 2016.

[17] S. Wu, M. Chen, G. Wu, and C. Zhou, "Variation of core-shell structural particles and their toughening behavior in poly (vinyl chloride) (PVC) matrix," Journal of Polymer Research, vol. 22, no. 5, 2015.

[18] J. Y. Qian, R. A. Pearson, V. L. Dimonie, O. L. Shaffer, and M. S. el-Aasser, "The role of dispersed phase morphology on toughening of epoxies," Polymer, vol. 38, no. 1, pp. 21-30, 1997.

[19] S. Guessasma, S. Belhabib, and H. Nouri, "Microstructure, thermal and mechanical behavior of 3D printed acrylonitrile styrene acrylate," Macromolecular Materials and Engineering, vol. 304, no. 7, article 1800793, 2019.

[20] W. Huang, Z. Mao, Z. Xu, B. Xiang, and J. Zhang, "Synthesis and characterization of size-tunable core-shell structural polyacrylate-graft-poly(acrylonitrile-ran-styrene) (ASA) by preemulsion semi- continuous polymerization," European Polymer Journal, vol. 120, article 109247, 2019.

[21] C. S. Chern, "Emulsion polymerization mechanisms and kinetics," Progress in Polymer Science, vol. 31, no. 5, pp. 443-486, 2006.

[22] T. Tian, Z. Zhang, and J. Zhang, "Influence of acrylic content in PBA-SAN on the mechanical properties of PBA-SAN and PBA-SAN/SAN blends," Journal of Vinyl \& Additive Technology, vol. 24, no. 3, pp. 262-267, 2018.
[23] P. Zhao and J. Zhang, "Effect of poly(butyl acrylate)-g-poly(styrene-co-acrylonitrile)'s core/shell ratio on mechanical and thermal properties of poly(butyl acrylate)-g-poly(styrene-coacrylonitrile)/ $\alpha$-methylstyrene-acrylonitrile binary blends," Journal of Macromolecular Science, Part B, vol. 57, no. 3, pp. 231-242, 2018.

[24] Y. Han, Z.-X. Tai, C. Zhou, M. Y. Zhang, H. X. Zhang, and F. Q. Liu, "Influence of blend composition on the mechanical properties and morphology of PC/ASA/SAN ternary blends," Polymer Bulletin, vol. 62, no. 6, pp. 855-866, 2009.

[25] L. Ren, Y. Li, M. Zhang, Y. Han, and H. Zhang, "Toughness, dynamic mechanical property, and morphology of polyvinylchloride/acrylonitrile-styrene-butyl acrylate blends," Journal of Vinyl \& Additive Technology, vol. 22, no. 1, pp. 43-50, 2016.

[26] K. P. Zhang, A. Hamza Bichi, and J. Q. Yang, "Effect of acrylonitrile styrene acrylate on mechanical, thermal and three-body abrasion behaviors of eucalyptus fiber reinforced polyvinyl chloride composite," Materials Research Express, vol. 8, no. 2, article $025305,2021$.

[27] S. Tolue, M. R. Moghbeli, and S. M. Ghafelebashi, "Preparation of ASA (acrylonitrile-styrene-acrylate) structural latexes via seeded emulsion polymerization," European Polymer Journal, vol. 45, no. 3, pp. 714-720, 2009.

[28] X. Zhang, Y. Cao, Q. Jiang, Y. Zhang, and W. Yang, "Preparation of cross-linked poly(methyl methacrylate) microspheres using an asymmetric cross-linker via dispersion polymerization and its application in light diffusers," Colloid and Polymer Science, vol. 298, no. 4-5, pp. 495-504, 2020.

[29] B. Zhang, X. Lv, A. Zhu, J. Zheng, Y. Yang, and Z. An, "Morphological stabilization of block copolymer worms using asymmetric cross-linkers during polymerization-induced self-assembly," Macromolecules, vol. 51, no. 8, pp. 27762784, 2018.

[30] D. C. Sundberg and Y. G. Durant, "Latex particle morphology, fundamental aspects: a review," Polymer Reaction Engineering, vol. 11, no. 3, pp. 379-432, 2003.

[31] H. R. Sheu, M. S. el-Aasser, and J. W. Vanderhoff, "Phase separation in polystyrene latex interpenetrating polymer networks," Journal of Polymer Science Part A: Polymer Chemistry, vol. 28, no. 3, pp. 629-651, 1990.

[32] Y. G. Durant, E. J. Sundberg, and D. C. Sundberg, "Effects of cross-linking on the morphology of structured latex particles .2. Experimental evidence for lightly cross-linked systems," Macromolecules, vol. 30, no. 4, pp. 1028-1032, 1997.

[33] M. M. Hassan, T. Takahashi, and K. Koyama, "Thermal stability, mechanical properties, impact strength, and uniaxial extensional rheology of reactive blends of PS and SBS polymers," Polymer Bulletin, vol. 76, no. 11, pp. 5537-5557, 2019.

[34] A. Kaim, "Terminal and penultimate reactivity ratios in the styrene-acrylonitrile free-radical copolymerization system in bulk," Journal of Macromolecular Science, Part A: Pure and Applied Chemistry, vol. A35, no. 4, pp. 577-588, 1998.

[35] Y. Furushima, M. Nakada, H. Takahashi, and K. Ishikiriyama, "Study of melting and crystallization behavior of polyacrylonitrile using ultrafast differential scanning calorimetry," Polymer, vol. 55, no. 13, pp. 3075-3081, 2014.

[36] Z. Mao and J. Zhang, "Largely improved the low temperature toughness of acrylonitrile-styrene-acrylate (ASA) resin: fabricated a core-shell structure of two elastomers through the 
differences of interfacial tensions," Applied Surface Science, vol. 444, pp. 345-354, 2018.

[37] X. Zhang and J. Zhang, "Effect of core-shell structures of acrylonitrile-styrene-acrylate (ASA) terpolymer on the properties of poly(vinyl chloride) (PVC)/ASA blends: miscibility, toughness, and heat resistance," Journal of Applied Polymer Science, vol. 135, no. 43, article 46839, 2018.

[38] G. Gao, J. Zhang, H. Yang, C. Zhou, and H. Zhang, "Deformation mechanism of polystyrene toughened with submicrometer monodisperse rubber particles," Polymer International, vol. 55, no. 11, pp. 1215-1221, 2006.

[39] C. B. Bucknall and D. R. Paul, "Notched impact behavior of polymer blends: part 1: new model for particle size dependence," Polymer, vol. 50, no. 23, pp. 5539-5548, 2009.

[40] B. P. Panda, S. Mohanty, and S. K. Nayak, "Mechanism of toughening in rubber toughened polyolefin-a review," Polymer-Plastics Technology and Engineering, vol. 54, no. 5, pp. 462-473, 2015.

[41] M. A. Dundar and G. S. Dhaliwal, "Investigation for impact behavior of acrylonitrile-butadiene-styrene amorphous thermoplastic," Polymer Testing, vol. 89, article 106624, 2020.

[42] J. Wang, X. Zhang, L. Jiang, and J. Qiao, "Advances in toughened polymer materials by structured rubber particles," Progress in Polymer Science, vol. 98, article 101160, 2019. 